\title{
Remark on the Application of Smith Predictor in Thickness Control System of the Rolling Mill \\ Xin $\mathrm{ZHAO}{ }^{1, a}$, Peiyan $\mathrm{ZHU}^{2, \mathrm{~b}}$
}

${ }^{1}$ School of Electronic communication Engineering, Hainan Tropical Marine University, Sanya, Hainan, 572022, China

${ }^{2}$ Liaoning Metallurgical Vocational and Technical College, Benxi, Liaoning, 117000, China

aemail: 565332301@qq.com, bemail: 1586197498@qq.com

Keywords: Smith Predictor; Pure Time-delay; PID Control; Thickness Control System

\begin{abstract}
According to the characteristics of the time lag of rolling mill thickness control system, it used Smith predictor to compensate measured hysteresis. And by the internal model design method, from the perspective of the design predictive control system, and the Smith predictor and PID control method for system effects were compared by means of simulation method.
\end{abstract}

\section{Introduction}

Because the mill thickness gauge is installed in a certain distance from the mill, therefore, thickness closed loop control system with pure lagging link, for the pure lagging, the conventional PID feedback control system cannot achieve a very good control effect[1]. In the current commonly used pure delay compensation method, compensation effect of Smith predictor is good; its application in the automatic control system can reduce the control index of the delay system [2].

\section{Pure Lagging Smith Predictive Controls}

\section{Principle of Smith Predictive control.}

Smith pure lag compensation model is shown in Figure 1, the Smith predictor and control object $G(\mathrm{~s})$ is parallel, the parallel equivalent control object is:

$$
\hat{G}(s)=G(s)+G_{0}(s)\left(1-e^{-\tau s}\right)=G_{0}(s)
$$

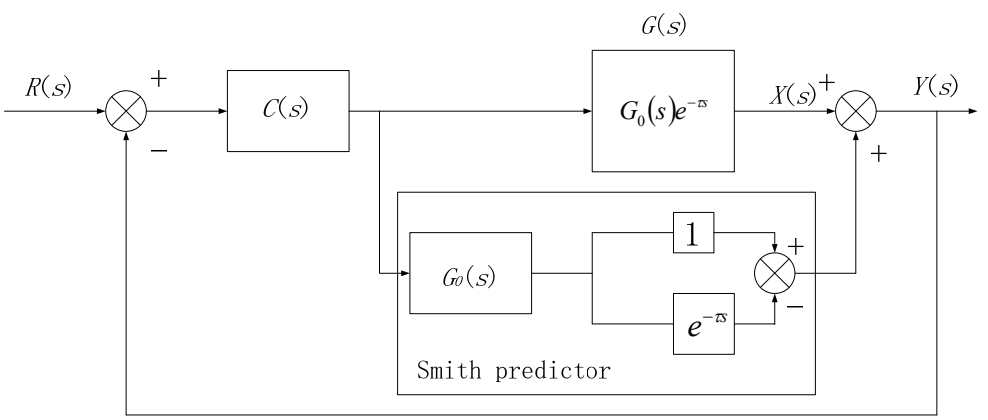

Fig. 1.Smith predictor model

$\mathrm{By}(1)$, it can be seen that the equivalent control object does not contain pure lag factor $e^{-\tau s}$, which makes the system design easier. From figure 1 the actual analysis shows that the relationship between the output variable $X(s)$ and the output $Y(s)$ of equivalent object is:

$$
Y(s)=X(s)+\frac{X(s)}{e^{-\tau s}}\left(1-e^{-\tau}\right) \quad=X(s) e^{\pi s}
$$

By (2), $X(s)$ is same as $Y(s)$, only $X(s)$ lag behind $Y(s)$ for $\tau$. So, as long as the output $Y(s)$ is stable, the actual output $X(s)$ will also be stable.

Essence of Smith Predicting Control.

From Fig.1, yield: 


$$
\frac{X(s)}{R(s)}=\frac{C(s) G(s)}{1+C(S) G(s) e^{\tau s}}
$$

The block diagram of actual control variable is shown as follows:

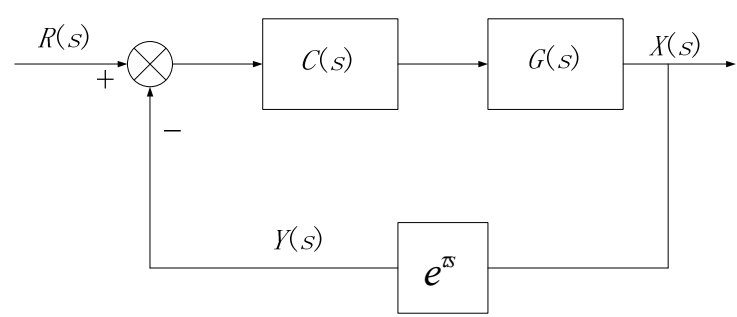

Fig.2.Another block diagram of predictive control system

From Figure 2, it can be seen clearly that Smith predicts substantially is equivalent to insert a prediction unit of $e^{\tau s}$ in a feedback loop to bring forward the output $Y(s)$ of equivalent control object for $\tau$, so as to offset the delay time $\tau$ for the control object. However, using hardware to realize the $e^{\tau s}$ is much harder than $e^{-\tau s}$, the benefits of Smith predictor is to avoid the effect of $e^{\tau s}$, using the predictor connected in parallel with the original object to achieve the effect of $e^{\tau s}$ objectively.

\section{Improvement Project of Smith Predictive Control.}

Although the Smith predictive control can be realized physically, but there are still some problems in application, especially in the case of internal and external disturbances, the robust stability is not high. Aiming at this problem, at present, many scholars at home and abroad has researched Smith predictive control system from two aspects of theory and structure and put forward many kinds of improvement scheme [3].

From the aspect of theory research, it is mainly studied from the analysis of the effect of model parameter mismatch on the Smith predictor robust, and then quantifies various model errors, then uses mathematical tools to analyze it qualitatively and quantitatively, so as to find out the improved method Smith predictive controller with robust control of loss of model.

From the structure, on the basis of Smith predictive control system, it is added an additional part to get a new Smith predictor, or a variety of other control algorithms are combined to extract some valuable compound control strategy, thereby increasing the system robustness to restrain disturbances.

In addition, the actual control system can be introduced into internal model design method to study the lag. The result shows that the Smith predictive system designed by internal model design method can improve dynamic indicators of the control system.

\section{Design Method of Internal Model}

Garaia and Morari proposed the internal model control in 1982, which advantage is able to induce PID control, Smith predictive control, Deterministic linear quadratic optimal feedback control and a variety of predictive control to the same framework [4], so the Smith predictive control can be regarded as a kind of special situation of the internal model control, we may wish to directly introduce the internal model (IMC) related design theory.

\section{Design Idea of Internal Model.}

Before discussing the internal model design method, we might as well review the commonly used open-loop control and closed-loop control system features.

As we know, for the open loop control, as long as improving the controller $C(s)$ and object $G(s)$ precision, then the output precision can be guaranteed. But its shortcoming is that it hasn't adjustment ability to object properties change or external disturbance. The closed loop control system can feedback the object properties change and disturbance. However, the feedback signal is taken directly from the output, which makes effect of the unpredictable interference $f$ on the output mixed together with other factors, and even the effects swamped by other amounts of factors and not been compensated timely, thus affecting the control effect. If we will transform it into internal model control structure, as shown in Figure 3, compared with the figure 1, it can be seen, with the structure of internal model control system, due to the introduction of the internal model, the 
feedback from the output feedback has been turned into perturbation feedback [5].

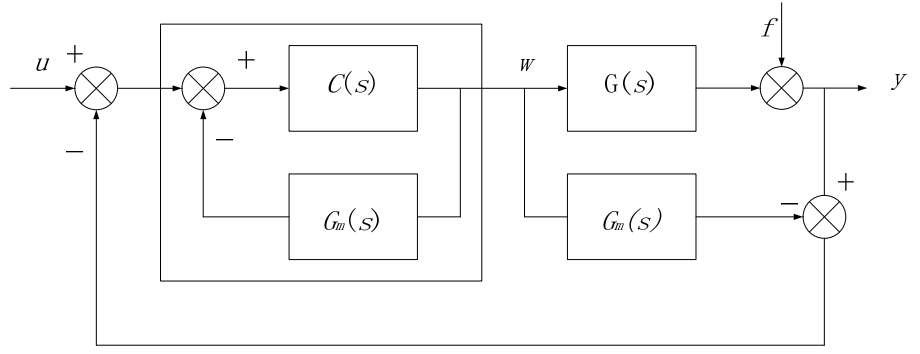

Fig.3.Equivalent internal model control

When the model $\mathrm{Gm}$ mismatch object $\mathrm{G}$, the feedback information, in addition to the original disturbance $\mathrm{f}$, also contains some mismatched information of model, anti-disturbance design which is beneficial to the control system, and increase the robustness of the system. And the controller design is also more easily, when the model matching $(\mathrm{Gm}=\mathrm{G})$, the closed loop system in figure 3 is equivalent to the open loop, which can be designed according to the method of open loop. Therefore, the internal model analysis method also has the advantages of open loop and closed loop, the design is also simple, relatively easy to obtain good dynamic response, and robust performance can be regarded as a design goal, is an effective design method.

\section{Structure of Improved Smith Predictor.}

For the thickness closed-loop control system, the Smith predictor based on internal model control structure is adopted, as shown in figure 4. In Figure 4, the part in the predictor which doesn't contain pure lagging link combined with controller C, $C(s)$ is a controller, $G(s)$ is the actual control object, $G_{m}(s)$ is a model, $G_{m 0}(s)$ is a part of the model which does not contain pure lagging part, $G_{f}(s)$ is a dynamic feedback link. Due to thickness gauge in the thickness closed loop control feedback loop is contained, it is a small inertia link, therefore, the structure of the Smith predictor structure adopted by the paper has a dynamic link $G_{f}(s)$ aim to traditional Smith, $G_{f}(s)=1 /\left(T_{h} s+1\right)$, $\mathrm{T}_{\mathrm{h}}$ is lagging time used to measure the thickness and data processing, here we can take $T_{h}=0.05 \mathrm{~s}$.

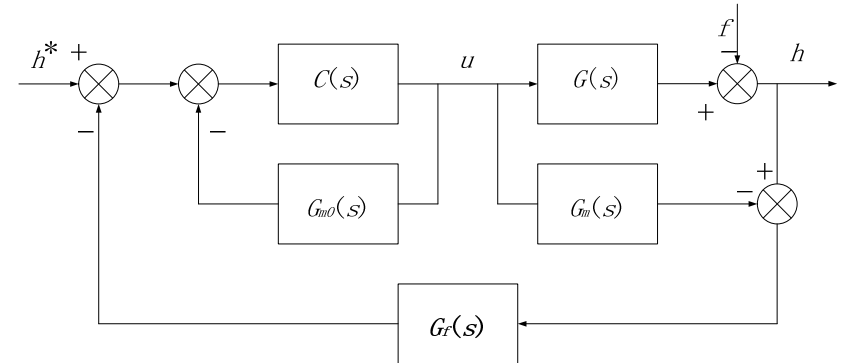

Fig.4. The block diagram of Smith predictor

The roll gap loop of rolling mill is studied as an example. That is, roll gap closed-loop is approximate rectified two order link, as follows:

$$
\phi_{p}(s)=-\frac{0.2 \omega_{P}{ }^{2}}{s^{2}+2 \xi_{P} \omega_{P} s+\omega_{P}{ }^{2}}
$$

Setting the parameters, $\xi_{P}=0.7, \omega_{P}=10 \mathrm{~s}^{-1}$, the dynamic characteristics of the position system on both sides is similar. This system uses the thickness gauge to measure the output thickness, the lag time is calculated according to $\tau_{\mathrm{P}}=L / V$, $\mathrm{L}$ is the distance between the mill center line and the thickness gauge, setting $L=1.6 \mathrm{~m}, V$ as the rolling speed, and then considering the rolling mill press efficiency, the transfer function of thickness closed-loop is

$$
G(s)=-\frac{0.2 K_{G} \omega_{P}^{2} e^{-\tau_{p} s}}{\left(K_{G}+W\right)\left(s^{2}+2 \xi_{P} s+\omega_{P}^{2}\right)}
$$

The transfer function of model is

$$
G_{m}(s)=-\frac{0.2 K_{m G} \omega_{m p}^{2} e^{-\tau_{m p} s}}{\left(K_{m G}+W_{m}\right)\left(s^{2}+2 \xi_{m p} \omega_{m p} s+\omega_{m p}^{2}\right)}
$$




$$
G_{m 0}(s)=-\frac{0.2 K_{m G} \omega_{m p}{ }^{2}}{\left(K_{m G}+W_{m}\right)\left(s^{2}+2 \xi_{m p} \omega_{m p} s+\omega_{m p}^{2}\right)}
$$

Here, $W_{m}$ is the Plastic stiffness coefficient; $K_{m G}$ is modulus of the rolling mill.

\section{Smith Predictor and PID Controller}

\section{Equivalent Parameters of Smith Predictor and PID Controller.}

The traditional PID controller cannot control pure lag object, and the Smith predictor can solve this problem well. Figure 5 shows a typical feedback control system.

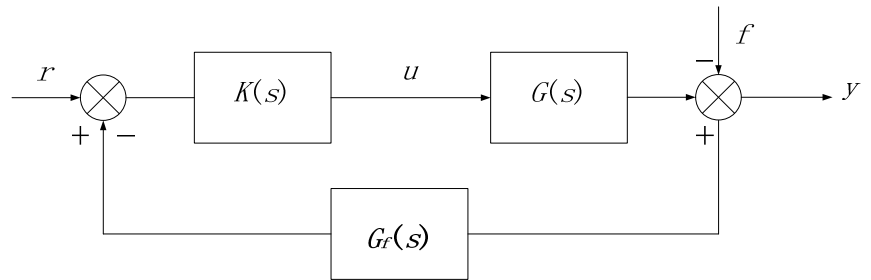

Fig.5.Typical feedback control system

Among them, $K(s)$ is the controller, $G(s)$ is the control object, $G_{f}(s)$ is a dynamic link loop feedback, compared with figure 1, substituting the data as before, yield:

$$
K(s)=-\frac{57(0.1914 s+1)\left(1+\frac{\tau_{m p}}{2} s\right)}{10 s\left(\lambda+\tau_{m p}+\frac{\tau_{m p} \lambda}{2} s\right)}
$$

$K(\mathrm{~s})$ structure is incomplete differential PID controller, $\tau_{m p}$ is the lag time in the model for the Smith estimate, which is changing with the rolling speed, $\lambda$ is the adjustable parameter.

Simulating Research.

In the rolling process, the lag time $\tau_{p}$ is changing. Using Simulink model to obtain the [6] data as follows:

\begin{tabular}{|c|c|c|c|c|c|c|c|}
\hline \multicolumn{8}{|c|}{ simulation data for ideal situation } \\
\hline \multicolumn{4}{|c|}{$\tau_{p}=0.16 \mathrm{~s}, \quad \lambda=0.2$} & \multicolumn{4}{|c|}{$\tau_{p}=4 \mathrm{~s}, \quad \lambda=2$} \\
\hline \multicolumn{2}{|c|}{ PID controller } & \multicolumn{2}{|c|}{ Smith predictor } & \multicolumn{2}{|c|}{ PID controller } & \multicolumn{2}{|c|}{ Smith predictor } \\
\hline overshoot & $\begin{array}{l}\text { Adjusting } \\
\text { time }\end{array}$ & overshoot & $\begin{array}{l}\text { Adjusting } \\
\text { time }\end{array}$ & overshoot & $\begin{array}{l}\text { Adjusting } \\
\text { time }\end{array}$ & overshoot & $\begin{array}{l}\text { Adjusting } \\
\text { time }\end{array}$ \\
\hline $20 \%$ & $2 \mathrm{~s}$ & $7 \%$ & $1 \mathrm{~s}$ & $8 \%$ & $16 \mathrm{~s}$ & 0 & $8 \mathrm{~s}$ \\
\hline \multicolumn{8}{|c|}{ simulation data for mismatched situation } \\
\hline \multicolumn{4}{|c|}{$\tau_{p}=0.26 \mathrm{~s}, \lambda=0.3$} & \multicolumn{4}{|c|}{$\tau_{p}=4 \mathrm{~s}, \quad \lambda=1$} \\
\hline \multicolumn{2}{|c|}{ PID controller } & \multicolumn{2}{|c|}{ Smith predictor } & \multicolumn{2}{|c|}{ PID controller } & \multicolumn{2}{|c|}{ Smith predictor } \\
\hline overshoot & $\begin{array}{l}\text { Adjusting } \\
\text { time }\end{array}$ & overshoot & $\begin{array}{l}\text { Adjusting } \\
\text { time }\end{array}$ & overshoot & $\begin{array}{l}\text { Adjusting } \\
\text { time }\end{array}$ & overshoot & $\begin{array}{l}\text { Adjusting } \\
\text { time }\end{array}$ \\
\hline $16 \%$ & $2 \mathrm{~s}$ & $14 \%$ & $1.5 \mathrm{~s}$ & $8 \%$ & $18 \mathrm{~s}$ & 0 & $8 \mathrm{~s}$ \\
\hline
\end{tabular}

Table 1 simulation data for Smith predictor and PID controller under equivalent parameters

The simulation waveform is as follows:

(1) ideal situation 


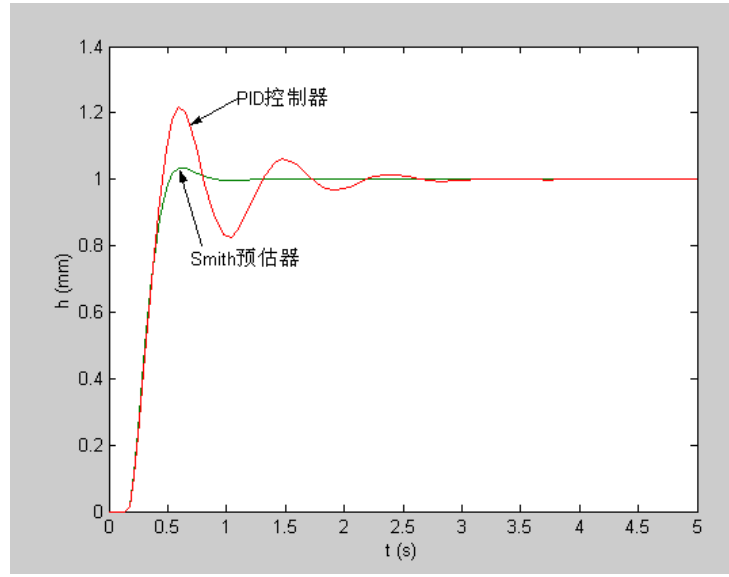

Fig.6.The step response at ligh speed of the system $\left(\tau_{p}=0.16 \mathrm{~s}, \lambda=0.2\right)$

(2) mismatched situation

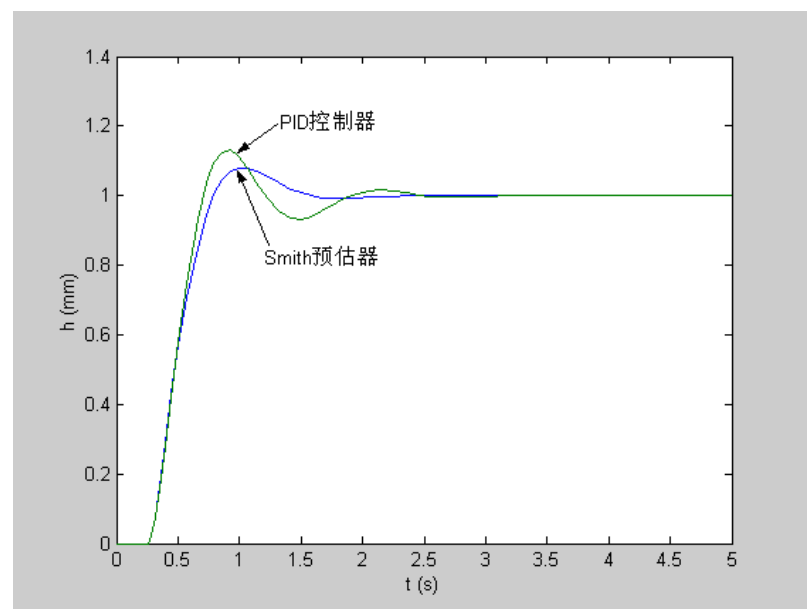

Fig.8.The step response at high speed of the system $\left(\tau_{p}=0.26 \mathrm{~s}, \lambda=0.3\right)$

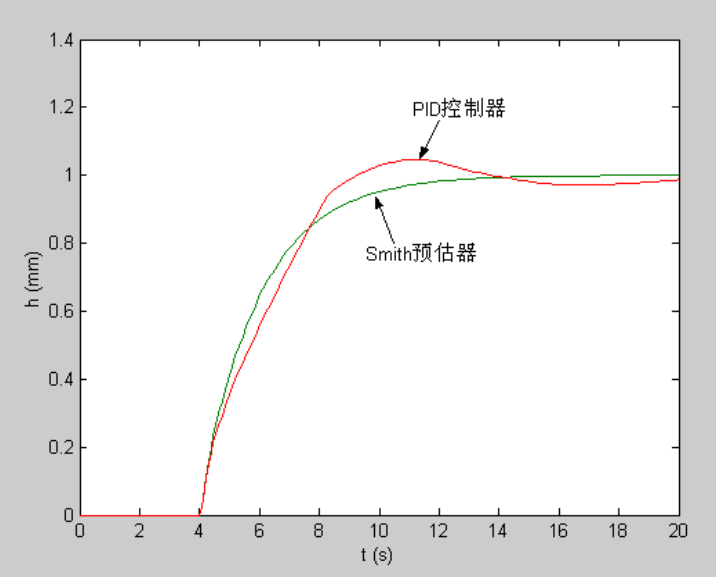

Fig.7.The step response at low speed of the system $\left(\tau_{p}=4 \mathrm{~s}, \lambda=2\right)$

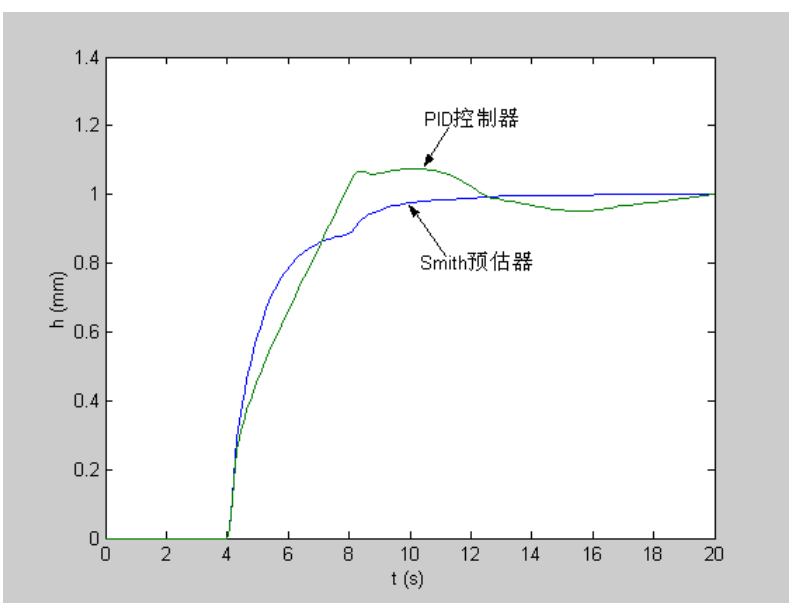

Fig.9.The step response at low speed of the system $\left(\tau_{p}=4 \mathrm{~s}, \lambda=1\right)$

\section{Conclusions}

According to pure delay link in the thickness closed-loop control system, it used Smith predictive compensation method to offset the measured hysteresis effect. Using internal model designed the Smith predictor, and the inner loop transfer function is equivalent to a typical two order the system, from simulation results it can be seen that Smith predictor controller is better than the traditional PID controller.

\section{References}

[1] Wu Hansheng. Adaptive Stabilizing State Feedback Controllers of Uncertain Dynamical Systems with Multiple Time Delay [J].IEEE Transactions on Automatic Control, 2000,45(9):1697-1701.

[2] Li Lizheng. Tuzzy Estimate Controlling of Pure Time-delay Process Control. Transactions of Traffic University of Changsha, 1996(2):14-18.

[3] Darko Vrecko, Damir Vrancic, Dani Jurici C, etal. A New Modified Smith Predictor: the Concept, Design and Tuning （J）.ISA Transactions, 2001, 40(2):111-121.

[4] Ray W H. Multivariable Process Control -A survey. Computer and Chemical Engineering, 
1988,7(2):367.

[5] Yang Jingming, Chao Zhiwen, Feng Yali etal. Innovative Approach of Thickness Control system in $1270 \mathrm{~mm}$ cold rolling mill on hydraulic pressure. Metallurgical industry automation, 2003,3(2):23-26.

[6] Xue Dingyu,Chen Yangquan. Simulation Technology of System and Application on MATLAB/Simulink. Publishing house Qinghua University, 2002. 